\title{
Interlaboratory comparisons of the Regional Metrological Organization in the field of Rockwell's scales (progress and perspectives)
}

\author{
V. Skliarov \\ National Scientific Centre “Institute of Metrology”, 42, Myronosytska Str., Kharkiv, 61002, Ukraine
}

\begin{abstract}
Résumé. À la lumière de la nouvelle définition Rockwell $\mathrm{C}$ de l'échelle de dureté adoptée par national de métrologie Instituts (INM) [1], une comparaison de la dureté Rockwell et superficialité Rockwell échelles réalisée par la dureté primaire machines nationales Rockwell est nécessaire de vérifier la mise en œuvre de la nouvelle définition. Un protocole technique d'une organisation métrologique comparaison régionale clé (KC) a été présenté et approuvé lors de la réunion t TC 1.6 en Septembre 2014, Braunschweig, Allemagne. Une comparaison clé régionale (RKC) a été choisi et le protocole technique a été préparé en suivant les règles données par le "document des comparaisons de mesure dans le CIPM MRA» [2].
\end{abstract}

\section{Introduction}

The aim of the regional key comparison is to extend the metrological equivalence over the measurement standards of national metrology institutes, which did not take part in CIPM KC [3].

The degree of equivalence of measurement standards of the NMIs participating in key comparisons of a Regional Metrology Organization (RMO), is determined in accordance with section T4 of the Technical Supplement to the Mutual Recognition Arrangement (MRA) with respect to a reference value of CIPM KCRV, using the results of measurements obtained at the NMIs that participated in both comparisons (linking NMIs).

The procedures used for RMO KC data evaluation is intended to provide linking to CIPM KC data with low uncertainty and they should correspond to those used for CIPM KC data evaluation.

The National Scientific Centre "Institute of Metrology" (NSC "IM") - pilot laboratory decided to purchase the test blocks required for the comparison from a single source to eliminate any question of a block bias due to using blocks from different manufacturers. The RMO KC requires each NMI to test 5 (five) hardness levels using their own indenter. The proposed 5 (five) hardness levels are 80-86 HRA, 80-100 HRBW, 20-30 HRC, 40-50 HRC and 60-70 HRC. It was determined that one set of blocks will be required for the Regional Key Comparison.

\section{Program of measurements}

For the RMO KC, there will be 1 set of hardness reference blocks of the Rockwell scale, consisting of 5 (five) blocks with the hardness levels 80-86 HRA, 80-100 HRBW, 20-30 HRC, 40-50 HRC, 60-70 HRC (Figure 1). Hardness blocks are manufactured by "Centre "MET" Ltd (Russia) and have a length of 60 $\mathrm{mm}$, width of $40 \mathrm{~mm}$ and a thickness of $6 \mathrm{~mm}$.

The upper surface of the block, which is the measurement surface, is finished. The measurement area is defined to be within an engraved grid. A grid $(7 \mathrm{~mm} \times 7 \mathrm{~mm})$ is engraved on the block surface in order to define the coordinates of the test locations. The sizes of the test area and grid allow 35 possible test locations (Figure 2).

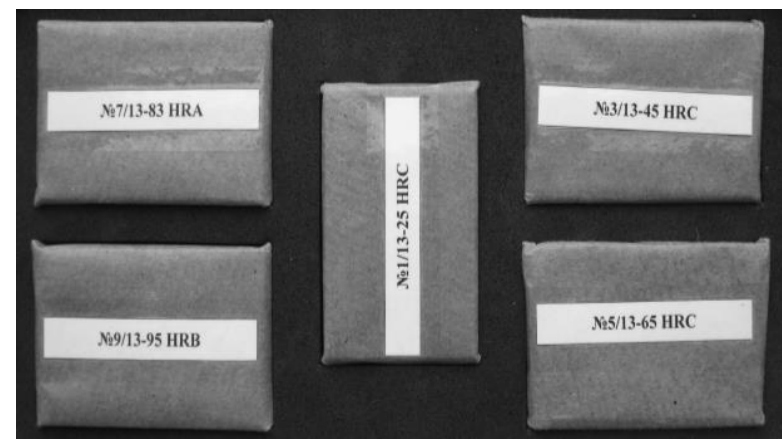

Figure 1. Rockwell set of hardness reference blocks 


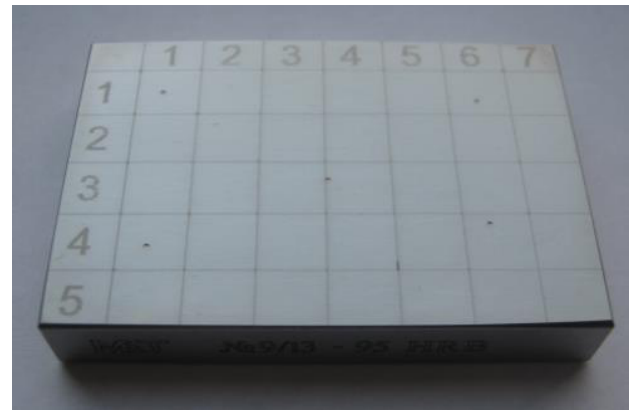

Figure 2. Layout of the grid on the measurement surface of the hardness reference blocks

The pilot laboratory are responsible for purchasing the blocks for their regional comparison, while each participating institute bears the costs for transport, customs and related administrative fees.

The pilot laboratory is to make measurements at the beginning and at the end of the RKC in order to evaluate the stability of the hardness reference blocks used in the RKC.

\section{Procedure}

The measurements shall be carried out following the Rockwell scale definition developed for adoption by National Metrology Institutes (NMIs). Any deviations from the procedure must be reported. Before conducting the measurements, each participant shall carry out the calibration of the primary hardness machine. Results of the calibration are:

- measurement results and uncertainties of the test forces $\left(F_{0}, u_{F 0}\right.$ and $\left.F, u_{F}\right)$,

- uncertainty of the length measuring system $u_{l}$, measurement results and uncertainties of the indenter geometry (plane angle $\alpha_{m}, u_{\alpha m}$, spherical tip radius $R_{a}, u_{R a}$ ),

- measurement results and uncertainties of the indenter geometry (spherical tip diameter $R_{\beta}, u_{R \beta}$ ),

- measurement result and uncertainty of the final indentation speed of additional load application $\left(V_{f i s}, u_{V f i s}\right)$,

- measurement results and uncertainties of the total time of preliminary test force $\left(T_{p}, u_{T p}\right)$,

- application time of preliminary test force $\left(T_{a}, u_{T a}\right)$,

- duration time of preliminary test force $\left(T_{p m}, u_{T p m}\right)$,

- duration of the total force $\left(T_{d f}, u_{T d f}\right)$,

- final reading time $\left(T_{r f}, u_{T r f}\right)$,

- measurement result and uncertainty of the temperature of test $\left(T, u_{T}\right)$.

Each participant shall make 5 (five) measurement indentations on each hardness reference block. The 5 (five) indentations will be used to evaluate the stochastic deviations occurring during the measurements, including the evaluation of the inhomogeinity of the hardness distribution across the test surface of the hardness reference blocks. The example of the test locations on the blocks provided to participants as grid coordinates (Figure 3).

\begin{tabular}{|c|c|c|c|c|c|c|c|}
\hline & 1 & 2 & 3 & 4 & 5 & 6 & 7 \\
\hline 1 & U & B & G & B & G & U & C \\
\hline 2 & C & U & K & C & K & B & G \\
\hline 3 & B & G & C & U & B & C & K \\
\hline 4 & U & K & B & G & K & U & G \\
\hline 5 & G & C & K & U & B & C & K \\
\hline
\end{tabular}

Figure 3. Test locations on the reference block

For each participant lab was determined symbol (see Table 1). It will be used by the participants to find the location of the indentations on the hardness block surface (1-5), as indicated in Figure 3. As additional locations (6 and 7), for test measurement or in case a measurement error occurs and the measurement must be repeated, the locations indicated as "repeat measurements" can be used.

Each indentation make at the center of the open square within the engraved grid lines. An indentation must not contact an engraved line. If a measurement error occurs, participant register the error in the data report in the field for measurement errors (designation "error") and repeat the measurement at one of the unused locations designated for repeat measurements.

After completing all measurements, participant must clean the blocks with alcohol, then wrap the blocks in anti-corrosion paper and put them in a plastic bag. The blocks are to be securely packed in the original shipping container and shipped to the next participant. When the blocks are shipped, the sending institute must again notify the pilot laboratory by fax or email.

Due to the number of fields on the hardness block surface (35) and the number of indentations to be carried out by each laboratory ( 5 plus two), the maximum number of participants for each regional comparison is $5(35 / 5=5+2$ for possible errors or test indentation).

\section{Time table of the measurements}

Table 1. Time table of the measurements

\begin{tabular}{|c|c|c|}
\hline $\begin{array}{c}\text { The symbol } \\
\text { of the cell }\end{array}$ & Institute/Country & Date \\
\hline $\mathbf{U}$ & NSC "IM", Ukraine & $\begin{array}{c}\text { November } \\
\mathbf{2 0 1 3}\end{array}$ \\
\hline $\mathbf{G}$ & PTB, Germany & $\begin{array}{c}\text { January } \\
\mathbf{2 0 1 4}\end{array}$ \\
\hline $\mathbf{C}$ & CMI, Czech Republic & $\begin{array}{c}\text { May } \\
\mathbf{2 0 1 4}\end{array}$ \\
\hline $\mathbf{B}$ & BelGIM, Belarus & $\begin{array}{c}\text { June } \\
\mathbf{2 0 1 4}\end{array}$ \\
\hline $\mathbf{K}$ & RSE "KazInMetr", & $\begin{array}{c}\text { December } \\
\mathbf{2 0 1 4}\end{array}$ \\
\hline $\mathbf{U}$ & Kazakhstan & $\begin{array}{c}\text { May } \\
\mathbf{2 0 1 5}\end{array}$ \\
\hline
\end{tabular}




\section{Uncertainty evaluation}

The Pilot laboratory must perform the measurements twice. After RSE "KazInMetr", re-indentation for the pilot laboratory will be performed in the free zones.

"A result from a participant is not considered complete without an associated uncertainty, and is not included in the draft report unless it is accompanied by an uncertainty supported by a complete uncertainty budget. Uncertainties are drawn up following the guidance given in the technical protocol" [2]. The uncertainty in measurement should be estimate according to the GUM [3,5]. At least the following influence quantities on the uncertainty should be considered:

- test forces;

- depth measuring system;

- cone angle of the indenter;

- spherical tip of the indenter;

- final indentation speed of additional force application;

- total time of preliminary test force;

- duration of the total force;

- final reading time;

- reproducibility of the primary hardness machine.

Each laboratory has the responsibility for determining their own uncertainty budget, including the determination of the sensitivity coefficients.

The draft of EURAMET/cg-16/v.01 [5,6] may be used. For the uncertainty budget calculation and for the sensitivity coefficients.

\section{Data compilation}

Pilot Laboratory is responsible for collecting and compiling the measurement data from participants.

The Draft A should be prepared as soon as all the results have been received from the participants. It includes the results transmitted by the participants, identified by name. It is confidential to the participants. After comments, remarks and discussion, the Draft B, should be subsequently prepared for the pilot laboratory and will include the Appendix containing proposals for a reference value and degrees of equivalence.

According to the $[6,7]$ we have two procedures of data evaluation designated by " $C$ " and " $D$ " which conventionally correspond to two types of comparison and ways for RMO $\mathrm{KC}$ data transformation.
The "C" procedure can be applied to those comparisons that require determination of a physical quantity value and assignment of this value to the material measure. In the appropriate CIPM and RMO key comparisons, the material measures with close but, nevertheless, different values of a physical quantity can be used as travelling standards. The " $\mathrm{C}$ " procedure requires application of an additive correction for RMO KC data. It is assumed that measurement uncertainties associated with the results of linking NMIs, obtained in the CIPM KC and RMO $\mathrm{KC}$, remain the same. It is our case. It is important to take into account that in comparisons take part institutions with CMC line in BIPM. The Draft B should be presented and discussed at the next meeting TC 1.6 for agreement. After agreement Draft $\mathrm{B}$ should be presented to CCM-WGH. If it is approved by the CCM-WGH, the Final Report will be prepared and sent to the CCM.

\section{Perspectives}

In carrying out comparisons on scales SuperficialRockwell use measures with a large number of zones for indentation. This will allow a greater number of participating NMI. Subject to availability on measures to give the status of comparisons «ongoing».

At presence of a second set of measures of hardness, with the participants agree on the use of the general indenter during comparisons SuperficialRockwell.

\section{Conclusion}

During international comparisons, the calculated values of the expanded uncertainty are compared with the values of the expanded uncertainty of the participating countries of international comparisons. For each sub band, the hardness are plotted showing the measured each participant comparisons hardness values with expanded uncertainty.

As an example, in Figure 4 shown a graphical representation of the measurement result for the subband 80-100 HRBW for a 5 NMI - comparisons of participants. According to the results of the graphical representation of the results it is concluded confirmation or non-confirmation of the declared values of uncertainty for each participant for each of the sub hardness. 


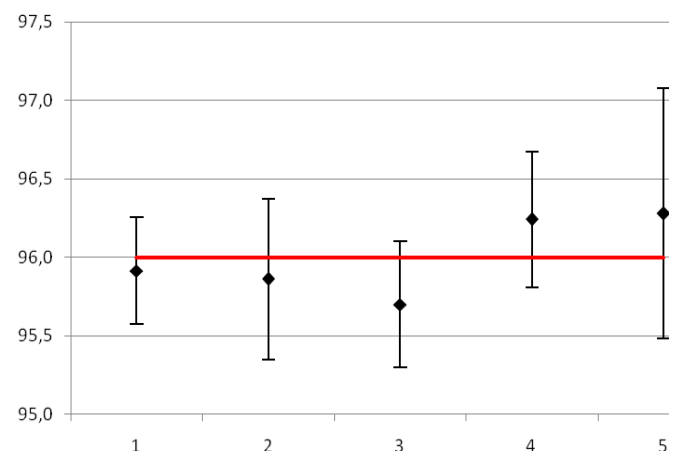

Figure 4. The results of international comparisons of the reference point 96,0 HRBW (as an example)

The positive results of international comparisons allow participating countries confirm the implementation of the "Agreement on mutual recognition of National measurement standards and calibration and measurement certificates issued by the National metrology institutes» and get the right to submit the appropriate line CMC (calibration and measurement capabilities) for calibration and measurement capabilities of the country on the website JCRB (Joint Committee of the Regional Metrology Organizations and the BIPM) - Joint Committee of the International Bureau of Weights and Measures (BIPM) and the RMO.

\section{References}

1. HRC Definition.

2. Measurement comparisons in the CIPM MRA.

3. Bureau International des Poids et Mesures. Mutual recognition of national standards and of calibration and measurement certificates issued by national metrology institutes BIPM Publication (1999)

4. JCGM 100:2008 (BIPM, IEC, IFCC, ILAC, ISO, IUPAC, IUPAP and OIML), GUM with minor corrections, Evaluation of measurement data Guide to the expression of uncertainty in measurement. (1995)

5. EURAMET/cg-16/v.01 (Previously EA-10/16), Guidelines on the Estimation of Uncertainty in Hardness Measurements,

(2007).

6. L. Brice, F. Davis, A. Crawshaw, Uncertainty in hardness measurement, NPL Report, (2003)

7. COOMET R/GM/14 Guidelines for data evaluation of COOMET key comparison (2006) 